\title{
Estrategia de expansión para la Parrilla del Ñato a través de las franquicias en la ciudad de Manta. Período 2019
}

\section{Expansion strategy for the Parrillada del Nato through the franchises in the city of Manta. Period 2019}

Ing. Gustavo Espinoza Delgado

Mgs. (c) Ivonne Elizabeth Paredes Chévez, Ing. CPA

Consultor Independiente, Ecuador

Mgs. Pablo Ricardo San Andrés Reyes, Ing.

Universidad Ecotec, Ecuador

Autor para correspondencia: gustavo-ed7@hotmail.com, psanandres@ecotec.edu.ec, ivonnelizabeth.paredes@gmail.com

Fecha de recepción: 01 de agosto de 2018 - Fecha de aceptación: 01 de diciembre de 2018

Resumen: La estrategia de expansión para la "Parrilla del Ñato" a través de franquicias en la ciudad de Manta, es un caso emprendedor de distribución de alimentos exclusivos a un sector promovido por el turismo. La investigación intenta descubrir el nivel de aceptación del emprendimiento en la ciudad de Manta, con el propósito de incrementar la participación de mercado e invertir en nuevos proyectos a largo plazo. Existen diferentes estrategias empresariales que los negocios pueden implementar para su expansión, es necesario evaluar con eficacia y eficiencia cada una de ellas, para evitar la incidencia negativa sobre el flujo de caja del negocio. Resulta necesario, estudiar la situación actual de la organización antes de tomar decisiones gerenciales o administrativas. La administración estratégica sugiere desarrollar un análisis de los factores internos o externos que afectan a las entidades, antes de implementar estrategias efectivas, por tal motivo en el presente caso de estudio se evalúa el estado actual del emprendimiento a través de instrumentos académicos, que permite al investigador viabilizar el éxito de la inversión en un nuevo sector estratégico. La "Parrilla del Nato" tiene la tendencia operativa y logística para ser más rentable, ya que mantiene un buen producto de carne a la parrilla adquirido con sus proveedores de confianza, además de conservar un servicio de atención de calidad.

Palabras claves: franquicias; emprendimiento; pymes; sector estratégico; expansión de negocios

Abstract: The expansion strategy for "Parrilla del Nato" through franchises in the city of Manta, is an entrepreneurial case of distribution of exclusive foods to a sector promoted by tourism. The research tries to discover the level of acceptance of the enterprise in the city of Manta, with the purpose of increasing the market share and investing in new long-term projects. There are different business tactics that firms can implement for it, but it is necessary to evaluate with effectiveness and efficiency each of them, otherwise it would have a negative impact on the company's cash flow. That's why, it is essential to study the current situation of the organization before making decisions, being these ones managerial or administrative. 
The strategic administration suggests developing an analysis of the internal or external factors that affect the companies, before implementing effective strategies, for this reason the present case study evaluates the current status of the enterprise through academic instruments, which allows the researcher to make viable the success of the investment in a new strategic sector. This company has the operative and logistical tendency to be more profitable, since it maintains a good grilled meat product purchased with its trusted suppliers, in addition to keep a good quality service.

Key words: franchises; enterprise; internal and external factors; strategic sector

\section{Introducción}

Frente a un entorno cambiante y competitivo, es imprescindible reorganizar el negocio. Detrás de un emprendimiento hay un emprendedor, con oportunidades para abrirse en mercados altamente competitivos.

Para un emprendedor, la creación de empresas ${ }^{1}$ como iniciación de un nuevo negocio tendrá menor riesgo si lo hace por franquicia ${ }^{2}$, porque cuenta con respaldo de la marca, posicionamiento, calidad, optimización en inventarios entre otros aspectos. La franquicia permite abrir redes de negocios con menor inversión con cada punto de venta.

Arenas, M. (2017) indica que un negocio es exitoso a través de franquicias porque tiene una diferenciación en el diseño, materiales, tecnología, innovación, desarrollo, atención a la necesidad del cliente, costo, calidad u otros. Lo que constituye una oportunidad para restaurantes y comidas rápidas como estrategia de competitividad y expansión.

Investigadores como Rodríguez, 2012; Escalante y García, 2010 tal como lo citó Arenas, M. (2017), indican que el origen del modelo de franquicia fue en Estados Unidos, logrando a través de este formato un mercado con mayor madurez y el más grande del mundo.

Según Khan como lo citó Solís, V. y González, M (2007), Estados Unidos es un modelo en cuanto a ventas a través de sus 100 mayores franquicias en el sector de Hotelería y Restaurantes, para el año 1997 lograron ventas de 117,9 billones de dólares. Según Tomos y Asociados SL citado por Solís, et al. (2007) en España representaron el 14.68\% de los ingresos del comercio minorista.

La existencia de franquicia en un negocio en sus etapas iniciales se argumenta en la escasez de recursos financieros y el de motivación y control para la expansión. (Solís, V. y Gonzáles, M., 2007)

Los autores anteriores citan a Oxenfeldt y Kelly, 1969 por el argumento de escasez de recursos, en el que indican que los empresarios utilizan la franquicias para acceder a determinados recursos, entre ellos el capital según Oxenfeldt y Thompson, 1969; Ozanne y Hunt,

\footnotetext{
1 "La creación de empresas es considerada una variable fundamental para el desarrollo económico, tal como lo han remarcado diversos organismos relevantes como la OECD, el World Economic Forum e investigaciones internacionales como el Global Entrepreneurship Monitor, GEM.” (Amoros, J. y Varela, C., p. 7)

2 "El sistema de franquicia se desarrolla de forma imparable y contribuye de manera importante a la modernización del comercio.” (Castro, D. y Cataluña, R, 2004, p. 71)
} 
1971; Caves y Murphy, 1976. Mientras que Combs y Ketchen, 1999 argumentan el objetivo de ampliar el negocio rápidamente. No obstante, Carney y Gedajilovic, 1991; Combs y Castrogiovanni, 1994 indican que los negocios necesitan crecer rápidamente para poder competir con las más grandes. Y posteriormente según Oxenfeldt y Kelly, 1969; Hunt, 1973, están de acuerdo con la recompra de los establecimientos que fueron franquiciados.

Los franquiciados invierten su patrimonio, son responsables y administradores de los establecimientos franquiciados, adquieren derechos y como arriesgan su capital, se concentrarán más en perseguir objetivos del negocio, maximizar la renta y lograr más riqueza. Autores citados por Solís, V. y Gonzáles, M., (2007) coinciden en ello tales como: Brickely y Dark, 1987; Brickley, Dark y Wisbach, 1991, Lewin Solomons, 1998; Perales y Vásquez, 2003; Caves y Murphy, 1976; Mathewson y Winter, 1985; Brickley y Dark, 1987; Norton, 1988; López, 2002. El capital de un empresario tiene menor riesgo, al iniciar un negocio a través de franquicia, el franquiciador pactará con ética el contrato con el franquiciado, la entidad crecerá rápidamente maximizando los beneficios, rentabilidad, ganancias e ingresos sostenibles por el posicionamiento de la marca a nivel nacional y con miras a la internacionalización de la franquicia.

El éxito de una organización se define por la capacidad de crecer en la mejora continua de todos los procesos involucrados en cada una de las actividades diarias, tanto administrativas como operativas. Una manera de obtener la mejora es cuando la misma organización aprende de su experiencia, y también de otras, esto sucede cuando planifica su futuro tomando en cuenta el entorno cambiante que la envuelve, y el conjunto de fortalezas y debilidades que la determinan.

Apoyarse en las fortalezas para superar las debilidades es, sin duda la mejor opción de cambio o mejora. La planificación de las estrategias es uno de los principales modos de conseguir beneficios para la organización. Esta permite realizar un diagnóstico del contexto interno y externo para posteriormente analizar y determinar las estrategias para que la organización logre sus objetivos de posicionamiento como franquicia en la mente de los consumidores.

La tendencia del restaurante se la considera limitada por el hecho de mantenerse un tiempo considerable en el mercado y no ha desatado aun un crecimiento estructural en los diferentes cantones de la costa y sierra ecuatoriana, estando en un sitial de demanda alto y no tienen franquicias como negocio de ampliación en el mercado de carnes a nivel nacional e internacional. El objetivo es el analizar las acciones necesarias para que la viabilidad de esparcimiento y distribución de franquicias sea la más adecuada, considerando los montos de inversión y el análisis del mercado objetivo, siendo un preámbulo subjetivo del restaurante a la parrilla.

La intención del caso investigativo es conocer la viabilidad de crear una franquicia de la "Parrilla del Ñato" en la ciudad de Manta. En la actualidad el negocio de carnes ofrece su servicio a más de 40.000 clientes diariamente, que acuden a diferentes lugares donde se ofrece carnes a la parrilla, siendo alrededor de más de 2000 clientes, que prefieren carnes a la parrilla en el Nato, este dato fue tomado de la concurrencia diaria de 3 locales ubicados en el centro, norte 
de Guayaquil y Samborondón. Actualmente, según página web indica que atiende a 50.000 personas en sus 6 locales.

La mayoría de consumidores se le habrá pasado por su mente, imaginar el éxito de esta parrilla y la razón de estancarse sólo en la ciudad de Guayaquil, por lo que se estudia la manera más factible de expandirse hacia Manta, se apertura una interrogante, pues en la actualidad la ciudad pasa por un proceso de reactivación económica, esta cualidad del mercado hace interesante la investigación. El negocio con sus años de experiencia y reconocimiento sólo se han concentrado en dicha ciudad. Por lo que despierta un interés para expandir el negocio mediante propuestas estratégicas a través de franquicias bajo una nueva modalidad de contrato.

Manta es una ciudad que ha tenido un crecimiento urbanístico, cultural y económico en los últimos años, esto además ha integrado el turismo como una de sus principales fuentes de desarrollo. Mediante el turismo se ha aumentado distintas clases de negocios como hoteles y restaurantes, y a pesar que es un lugar donde predomina la comida de mariscos, los mantenses y sus visitantes por lo general mantienen una dieta balanceada e integran variedades de alimentos, entre ellas carne en todas sus presentaciones. Hoy en día existen restaurantes o parrillas de carnes, pero aún no existe una parrilla con estándares de calidad en la preparación, instalaciones adecuadas y atención de calidad como las brinda la Parrilla del Ñato, pues cuenta con una trayectoria en Guayaquil.

\section{Metodología}

Se aplicará un tipo de investigación exploratoria, ya que a través de las encuestas se puede captar información de los moradores aledaños al sector investigado, fortaleciendo las estrategias a implementar. Según Fidias G. Arias (2006) la define como "Aquella que se efectúa sobre un tema u objeto desconocido o poco estudiado, por lo que sus resultados constituyen una visión aproximado de dicho objeto, es decir, un nivel superficial de conocimientos"

Se desarrollará un estudio descriptivo, con detalle de la situación actual de la organización. Este procedimiento "consiste en la caracterización, hecho, fenómeno, individuo o grupo, con el fin de establecer su estructura o comportamiento. Los resultados de este tipo de investigación se ubican en un nivel intermedio en cuanto a la profundidad de los conocimientos se refiere". (Arias. F. 2006). Mediante esta técnica se determinará la información que permitirá entrar a una descripción mucho más amplia de aquellas conductas que se suscitan en la empresa, teniendo como énfasis de forma directa la conducta de los competidores directos e indirectos, esto será un indicador profundo de rentabilidad y liquidez para la Parrilla del Nato.

Se analizará a los clientes potenciales de Manta por medio de encuestas, éstas "A diferencia de un censo, donde todos los miembros de la población son estudiados, recogen información de una porción de la población de interés, dependiendo el tamaño de la muestra en el propósito del estudio. La información es recogida usando procedimientos estandarizados, a cada individuo se le hacen las mismas preguntas. La intención de la encuesta no es describir los individuos particulares quienes, por azar, son parte de la muestra sino obtener un perfil compuesto de la población”. (Rivero, 2008). Este estudio cuantitativo permitirá descifrar el conocimiento del servicio de alimentación que ofrece La parrilla del Nato y hallar el nivel de 
viabilidad de incursionar la franquicia en la ciudad de Manta. El punto clave de la investigación es obtener resultados que determinen la aceptación y preferencias de los consumidores.

Para calcular la cantidad a encuestar se aplicó el método estadístico del intervalo de confianza. "Manta, cuenta con una población aproximada de 242.000 habitantes", (INEC, 2011), pero se tomará como base de estudio 61.869 habitantes que forman parte de la población económicamente activa que labora en la zona urbana. Por ende, al aplicar la fórmula de la población finita da como resultado 382 personas a encuestar, que dentro del mismo se realizara un análisis e interpretación de resultados de acuerdo a cada una de las interrogantes planteadas. Es importante recalcar que se consideró un $95 \%$ de confianza, $50 \%$ de éxito y $50 \%$ de fracaso.

\section{Cálculo del tamaño de la muestra}

\section{Tabla 1}

\begin{tabular}{lr}
\hline \multicolumn{2}{c}{ Población Finita } \\
\hline $\mathrm{N}$ & 61869 \\
$\mathrm{Z}(95 \%$ confianza) & 1,96 \\
$\mathrm{P}$ & 0,5 \\
$\mathrm{Q}$ & 0,5 \\
$\mathrm{D}$ & 0,05 \\
\hline
\end{tabular}

Elaborado por: Ing. Gustavo Espinoza Delgado y Mgs. Pablo Ricardo San Andrés Reyes

$$
\mathrm{n}=\left(\mathrm{N} * \mathrm{Z}^{\wedge} 2 * \mathrm{p} * \mathrm{q}\right) /\left(\mathrm{d}^{\wedge} 2 *(\mathrm{~N}-1)+\mathrm{Z}^{\wedge} 2 * \mathrm{p} * \mathrm{q}\right)
$$

Donde:

$\mathrm{N}$ es la población total (61869)

$\mathrm{Z}$ el nivel de confianza seleccionado (1.96)

p la probabilidad de éxito $(0,50)$

q la probabilidad de fracaso $(0,50)$

$\mathrm{d}$ el margen de error permitido en el ejercicio $(0,05)$

$\mathrm{n}=\left(61869 * 1.96^{\wedge} 2 * 0.50 * 0.50\right) /\left(0.05^{\wedge} 2 *(61869-1)+1.96^{\wedge} 2 * 0.50 * 0.50\right)$

$\mathrm{n}=61869 * 3.8416 * 0.50 * 0.50 / 154.67+0.9604$

$\mathrm{n}=382$

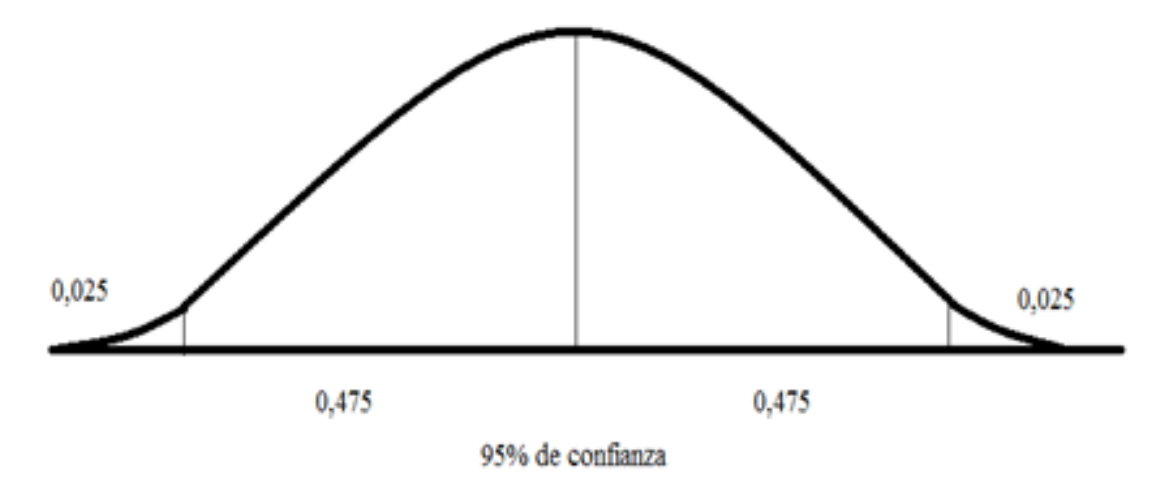


Figura 1

$$
100 \%-95 \%=5 \% \quad 0,05 / 2=0,025
$$

El método de la observación directa también se aplicó en la investigación, para determinar una cantidad demandada promedio de carnes a la parrilla de la organización. Por último, para evaluar la situación actual de la organización, se analizó las cinco fuerzas de Porter y los factores externos que podrían incidir directamente en los ingresos de la empresa, ya que antes de implementar estrategias empresariales es fundamental que los empresarios examinen criterios exógenos para tomar decisiones eficientes.

\section{Desarrollo}

\section{Franquicias como opción para la rápida expansión de negocios}

La franquicia como ampliación del negocio, consiguen un crecimiento superior de aquellas que no la emplean. (Solís, V. y Gonzáles, M., 2007)

Uno de los mayores problemas a la hora de expandirse es contar con los recursos pero eso puede hacerse con el dinero de los franquiciados, así como la pérdida entre control y administración se soluciona con las cláusulas para un acuerdo entre las partes y los costos por control de la red de negocios.

Lafontaine tal como lo citó Solís, V. y Gonzáles, M., (2007, p. 2753) piensa que "Las empresas que utilizan la franquicia cuando quieren crecer más rápido permite a los franquiciadores disminuir las restricciones a su crecimiento".

Fernández, et al., (2005) considera que para lograr el éxito se necesita una competencia con recursos estratégicos con conocimientos valiosos, primero en el mercado doméstico y luego buscará oportunidades a mercados internacionales. Según Harris y Cols, 1994; Donckles y Lambrecht, 1999 indican que los negocios familiares crecen despacio, porque suelen tener aversión al riesgo debido a que su patrimonio como riqueza familiar está comprometida, concuerda Wright y Cols, 1996.

La franquicia ayuda al reconocimiento de la marca, creación de cultura corporativa, prestigio. Negocios familiares con accionistas externos mejoran sus recursos, capacidad tecnológica, canales de distribución, comparten gastos y con ello logran el éxito nacional e internacional. (Fernández, et al., 2005)

La franquicia como estrategia empresarial según Vílchez, 2003; Prieto, Burgos, García y Rincón, 2016 como se citó en Arenas, M. (2017) tiene como objetivos:

1. Expansión de la empresa en el mercado

2. Búsqueda de socios

3. Sistema de cooperación 
4. Compartir riesgos

5. Ampliación de capacidad financiera

6. Apertura de mercados para mejor posicionamiento a nivel competitivo

7. Asegurar lugar en economía globalizada.

Quiroga como lo menciona Arenas, M. (2017) indica que la reglamentación de franquicias es a través de contrato, con elementos esenciales como el derecho de propiedad intelectual, Know- $\mathrm{How}^{3}$, asistencia técnica y contable y el precio.

Las franquicias generan mayor crecimiento ${ }^{4}$, expansión empresarial, productividad, competitividad, menores riesgos, posicionamiento, identidad corporativa, responsabilidad y costos compartidos, son buenos prospectos de negocios para cadena de restaurantes y comidas rápidas. (Arenas, M., 2017)

Castro, D. y Cataluña, R (2004) señalan razones para franquiciar no sólo por el conocimiento de la fórmula, sino también por motivos financieros, manejo del personal, conocimiento del negocio. Menciona a Vallet, quien indica que la franquicia es necesaria como estrategia para penetrar progresivamente el mercado, de forma más rentable, saturando el mercado nacional y logrando una expansión a nivel internacional.

Desventajas de la Franquicia: Cláusulas en el contrato que van en contra de libre competencia tales como imposiciones de precios, exclusividad territorial, de distribución, cánones, requerimientos. Por eso algunos países tienen oposición social como las asociaciones de consumidores, por contratos desequilibrados en favor únicamente del franquiciador. Sin embargo, eso se contrarresta con manejo ético y adecuado (Castro, D. y Cataluña, R).

Otra desventaja de expandir por franquicias una entidad es porque corresponde al negocio familiar. Como se citó en Fernández, Z \& Nieto, M (2005) existen pocos estudios disponibles en las Pymes ${ }^{5}$ de propiedad familiar según Gallo y García-Pont, 1996; Okorafo, 1999; Davis y Harveston, 2000. Según Ward (2008) la falta de recursos financieros, resistencia al cambio de los líderes familiares, diferentes objetivos y conflictos con los sucesores son límites al crecimiento del negocio.

Prieto y Villa como lo indicó Arenas, M. (2017), afirman que es necesario implementar acciones estratégicas con buenas relaciones entre franquiciador y franquiciado para una posición consolidada en el mercado.

Hay que tomar en consideración modelos de selección de punto de venta, modelos de atracción comercial, sistemas de información geográficas, para la localización óptima de unidades franquiciadas (Castro, D. \& Cataluña, R, 2004). No obstante, cuando un franquiciador

\footnotetext{
${ }^{3}$ Know How: saber hacer, secreto empresarial según Quiroga, 2012 como lo citó Arenas, M., (2017, p.164)

4 "El crecimiento es importante para las empresas, así como para la sociedad porque genera bienestar al proporcionar ingresos sostenibles". (Amoros, J. y Varela, C., p. 9)

5 "El crecimiento de las Pymes depende en gran medida de la capacidad empresarial y de una buena gestión de los recursos”. (Amoros, J. y Varela, C., p. 10)
} 
coloca el negocio y su marca está posicionada en el mercado hace que la ubicación geográfica del punto de venta sea menos atractiva para la competencia, generando una ventaja.

\section{Estudios de franquicias en países desarrollados y en vías de desarrollo}

En países desarrollados, la existencia de franquicias tiene ventaja sobre negocios independientes. Ayling como se citó en Castro, D. y Cataluña, R (2004) llega a concluir que en Reino Unido un negocio por franquicia en un período de 10 años es $80 \%$ frente a uno pequeño independiente.

Un estudio español logró demostrar el crecimiento de empresas pymes a través de franquicias, período 1996-2002, los resultados mostraron que las cadenas que utilizan la franquicia consiguen mayor crecimiento y de manera más rápida. (Solís, V. y Gonzáles, M., 2007). La franquicia es necesaria para que el pequeño y mediano negocio puedan expandirse, encontrar el capital de los franquiciados para la expansión y empleados que estén comprometidos con el negocio.

Gámir y Méndez como lo citó en Castro, D. y Cataluña, R (2004) realizaron una investigación en España señalando inconvenientes sociales de la franquicia. La mayor parte de las franquicias se localizan en grandes concentraciones de la urbe con presencia muy poco significativa en áreas lejanas a las mismas.

Según Arenas Marlly (2017) realizó un estudio en Colombia, con investigación descriptiva a una muestra de 877 restaurantes locales, que marcan tendencia en la franquicia como estrategia de prospecto de negocios debido a la creciente demanda de los consumidores. Sirgado y Lamas (2011) señala que es por la prioridad del tiempo y aceleración del ritmo de vida y poco tiempo disponible para comer por los horarios laborales.

El documento $\mathrm{PPES}^{6}$, indica que el mercado colombiano no se ha desarrollado lo suficiente, con 300 redes de 6,242 franquicias, en un mercado emergente, hay espacio para más negocios, no obstante Bogotá tiene el 36\% de participación en franquicias, Medellín 22\%, Cali $11 \%$ y resto de ciudades en un $31 \%$.

Según OCEX ${ }^{7}, 2016$ indica que en Colombia durante el año 2014 se abrieron cerca de 5 000 restaurantes, 580 franquicias, el $36 \%$ pertenecen al sector comidas.

Armando Kiyán de Perú fundador de "Pollos y Parrilladas Roky’s" una exitosa y ya internacional cadena de comida. Creció por la buena calidad y precios, actualmente cuenta con 40 locales a nivel nacional y 3 en Bolivia. (Amoros, J. y Varela, C., 2011)

\section{Manta y la competitividad}

\footnotetext{
6 “Programa de Promoción de Exportación de Servicios, (2004)," de la Comisión de la Promoción de Perú para la exportación y el Turismo, Promperú, documento que señala a la franquicia en Colombia como un negocio en auge.

${ }^{7}$ OCEX: Oficinas Comerciales del Perú en el Exterior
} 
Manta, es una ciudad con desarrollo urbanístico, cultural y económico. En los últimos años se ha incrementado el turismo como una de sus principales fuentes de desarrollo. Mediante este sector se ha desarrollado distintas clases de negocios como hoteles y restaurantes, a pesar que es un lugar donde predomina la comida de mariscos, los mantenses y sus visitantes desean otras variedades de comida como un buen corte de carne.

Hoy en día, existen restaurantes o parrillas de carnes, pero aún no existe una parrilla como la del Ñato con una trayectoria de cortes al estilo uruguayo y deseado como lo es en Guayaquil. Debido a ello se abre una interrogante que hace interesante la investigación y atrayente a un análisis de estrategias de expansión.

La competencia potencial para la parrilla del Ñato son todos aquellos establecimientos como es el Caminito, el Resero, Malbec, Marhambu, Martinica y Steak House que brindan cualquier tipo de comida, en especial parrilladas, dado que cualquier cliente puede ir a consumir en estos tipos de restaurantes. Existen en la actualidad muchos lugares que brindan variedades de carnes de todo tipo con sus respectivos derivados, los cuales se hacen útil al momento de preparar comidas, parrilladas y demás, entre los más destacados se menciona al "Chamo", el cual queda cerca del Mercado Central, en este local se ofrece variedades de carnes, embutidos y demás, sus precios son accesibles y de buena calidad, por otro lado, la comercializadora "El Chonero", que es muy conocida por su nivel de concurrencia de personas y la adquisición que estos presentan.

De igual forma "La casa de las carnes" ofrece a su clientela carnes para parrilladas siendo una de las más selectivas al momento de su venta y respectivas estrategias, Además, se encuentra la denominada "Hacienda" la cual otorga clasificaciones de carnes para todo tipo de ocasión, no obstante, dentro de estas resaltan las tres más importantes como lo viene siendo "El Mercado Central, "Mi Comisariato" y "Supermaxi", que son una de supermercados donde más abunda la comercialización y venta de productos tanto para parrillada como para todo tipo de platos, favoreciendo así a muchos de los locales que se caracterizan por la venta de comidas rápidas y a la carta como en este caso lo realiza la "Parrilla del Ñato".

Por otra parte, existen en la actualidad un sin número de competidores que ofrecen una variedad de platos exquisitos dentro de la rama de las parrilladas y platos a la carta, dentro de los mismos se destacan unos cuantos locales como competidores fuertes, los cuales vienen siendo el local "Beach Comber" siendo un local que brinda almuerzos y platos a la carta con sus respectivas carnes a la parrilla, la cual se vuelve una de los más fuertes para la Parrilla del Ñato, mientras que el local denominada "JUVA" es otro de los que ofrece variedades de platos relacionada con la parrillada y sus derivados, y la "Mamá Rosa" viene siendo una de los locales que ha crecido con el transcurso de los años de acuerdo a su alta gama de variedad de platos, volviéndose uno de los más amplios locales.

Mientras que para la Parrillada del Chamo se vuelve uno de los locales con más ventaja, puesto que, aparte de ser un local de venta de platos a la parrillada, es también uno de los distribuidores de carnes en el país, volviéndose uno de los más fuertes dentro del ámbito de comercialización y venta de comidas. Sin embargo, esta investigación que se realiza es para llegar a puntos de expansiones para que las estrategias de ventas sean atractivas y ayuden a 
beneficiar cada uno de los locales que tiene la Parrilla del Ñato, principalmente con la idea del local de apertura en la ciudad de Manta, haciendo que muchos de los franquiciadores deseen incursionar con el mismo nombre y variedad de platos a muchos lugares y cantones del país, volviéndose una de las más influyentes en la venta y comercialización en degustaciones, y consumo de carnes y embutidos de todo tipo, mismo que se beneficiará con estrategias de ventas factibles hacia los consumidores.

En la investigación se analizó las cinco fueras de Porter para determinar el nivel de rivalidad de los competidores directos, indirectos y productos sustitutos. Además, el poder de negociación de los proveedores y clientes.

\section{Rivalidad entre empresas competidoras}

En Manta aún no se han determinado extensamente restaurantes que vendan especialmente comida a la parrilla al estilo argentino, ya que en esta ciudad existen varios restaurantes como es el Resero, el Caminito, Malbec entre otros que preparan parrillas, pero de una manera tradicional, considerando la temática de la competencia, la infraestructura, productos y la calidad de servicio se considera que la competencia es "Media", pues a pesar que la parrilla del Nato cuenta con platos a la parrilla únicos existe algunos de lugares con productos similares. Es por este motivo que la parrillada quiere ingresar en el mercado de Manta con una nueva alternativa de inversión novedosa. El proyecto en sí, plantea la instalación de un restaurante, cuyo plato fuerte son las parrillas además de ofrecer platos típicos, los mismos que son preparados por chefs especialmente capacitados.

\section{Entrada potencial de nuevos competidores}

No se encuentran mayores obstáculos de entrada potenciales de nuevos competidores, esto hace que el ingreso al mercado de Manta no sea dificultoso. Pero es de suma importancia recalcar que al ser una nueva propuesta para la ciudad de Manta, muestra inseguridad en los inversionistas, lo que da un determinado tiempo para fortalecer el negocio.

\section{Presión de productos sustitutos}

Uno de las particularidades es el estilo argentino el cual es representar la rivalidad con otros productos ya posicionados en el mercado, entre los que se acentúan especialmente los asados y los platos típicos que ofrecen en la ciudad de Manta, por tal motivo se puede decir que no tiene una competencia directa. En la ciudad de Manta se encuentran varios restaurantes, de los cuales no poseen el debido reconocimiento. Constan también restaurantes que preparan parrillas como Choclo loco, Parrillada Jossy, y parrillas el Colorado, los mismos que se mantienen como baja competencia en el ámbito de alimentación de este sector, lo cual se caracteriza entre estos tipos de restaurantes que simplemente cuentan con una sola área de atención y lejos de la percepción de lo que sería un establecimiento de preparación de parrilladas. El nivel de rivalidad de productos sustitutos es alta debido a la gran variedad que esta ofrece.

\section{Poder de negociación de los proveedores}


Se refiere a propuesta de negociación con futuros proveedores. Se estima, además, que la carne, pollo, vegetales y demás ingredientes serían adquiridos en mercados como el Mercado Municipal de Manta, y demás supermercados de la ciudad. El poder de negociación de los proveedores es baja, pues se podría cambiar los insumos porque existen muchos proveedores potenciales y los costos de insumo no tienen influencia en los costos totales.

\section{Poder de negociación con los consumidores}

En este caso es restringido, porque brindan un servicio característico del resto restaurantes. Los clientes poseen poder de negociación media pues existen competidores secundarios que ofrecen parrilladas como: Asadero Solo Futbol, parrilladas El Colorado, Choclo Loco, que cuentan con precios más cómodos pero la debilidad de estos negocios es no contar con un estándar de calidad y procesos en sus actividades de producción.

La decisión que inclina a los consumidores obtener los servicios que el restaurante brinda se deriva en su generalidad, la calidad del servicio ofrecido, añadiéndole las ventajas cualitativas y cuantitativas perfeccionan la exigencia del cliente.

Con el propósito de complementar el análisis de las cinco fuerzas de Porter, se desarrolló un análisis de los factores externos que inciden en el negocio. En el ambiente político y legal se establece una extensión de la franquicia para inversionistas, que buscan un negocio rentable, donde las garantías se establecen en base a las políticas y logísticas manifestadas por el restaurante en las ciudades principales del Ecuador. Régimen antimonopolio apoya la expansión de los negocios sin generar el control mayoritario del mercado. La Ley del Comercio Exterior cuenta con estatutos que facilitan la importación de alimentos cárnicos que cuenten con registros sanitarios y certificaciones internacionales que avalen su consumo. Dentro de estas se regirán cada uno de los estatutos que se deben de cumplir en las expansiones y potestad de franquicias para el restaurante "La Parrilla del Nato", dando a conocer cada uno de los cumplimientos y detalle de las mismas.

Los factores tecnológicos reflejan la innovación y diferenciación en el servicio en donde se establece la aplicación de variedades de productos tecnológicos que permiten ofrecer un servicio adecuado, siendo necesario en primera instancia la seguridad y luego el servicio dotando al restaurante de sofisticados equipos de seguridad, cámaras, videos, proyecciones, sistemas multimedia, entre otros. Además, en el área de cocina está dotado de instrumentos tecnológicos que permiten ampliar la calidad de los platos que se elaboran a la parrilla.

A pesar de las políticas macroeconómicas ineficientes tales como: incremento de impuestos e inserción de barreras arancelarias por parte del gobierno en los últimos años, la organización ha podido mantener su liquidez en el tiempo.

\section{Resultados}

1. ¿Suele acudir a restaurantes en familia? 


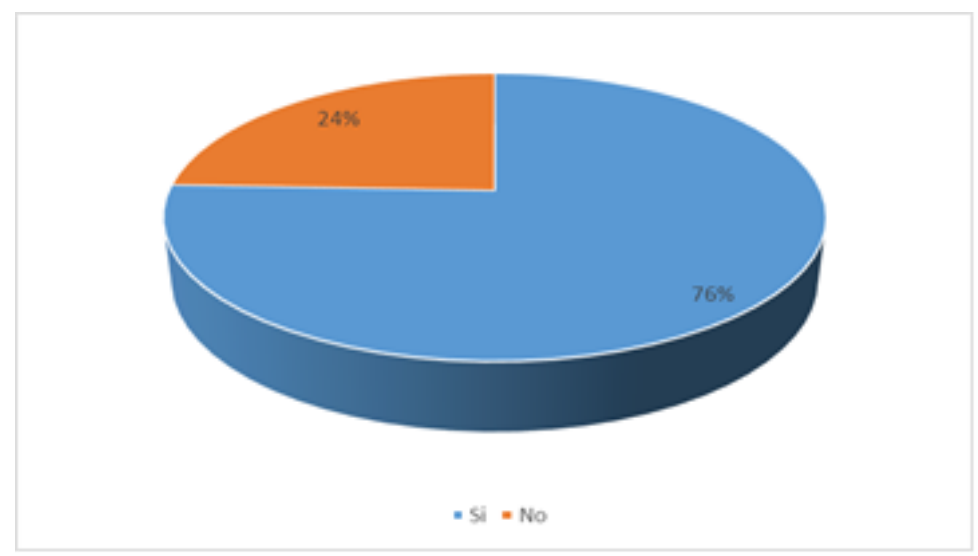

Figura 2. Asistencia a restaurantes en familia

Elaborado por: Ing. Gustavo Espinoza Delgado y Mgs. Pablo Ricardo San Andrés Reyes

El 76\% de los encuestados señalaron que acuden a un restaurante con su familia, mientras que el $24 \%$ mencionaron que suelen asistir solos.

2. ¿Qué tipo de comida prefiere usted en restaurantes?

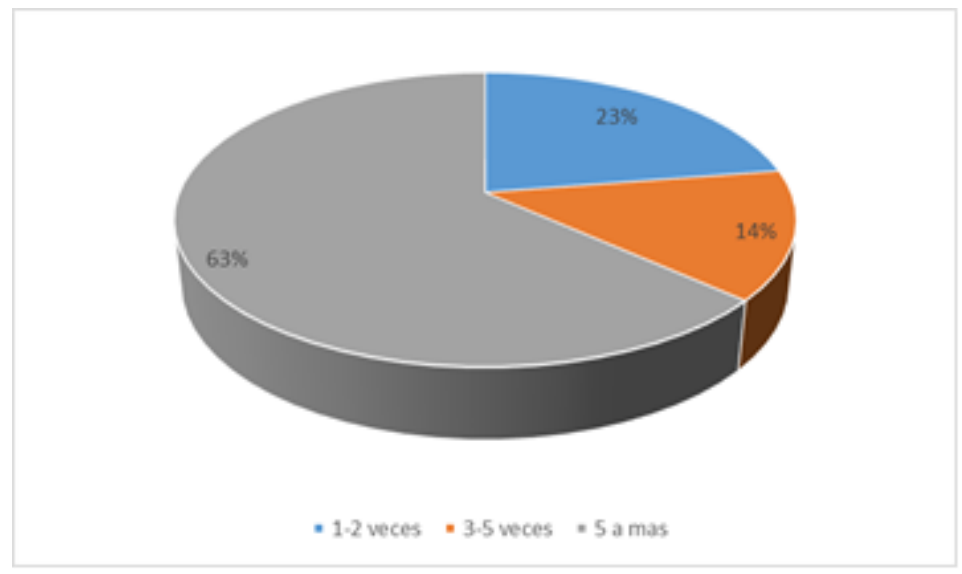

Figura 3. Gustos y preferencias en restaurantes

Elaborado por: Ing. Gustavo Espinoza Delgado y Mgs. Pablo Ricardo San Andrés Reyes

El $27 \%$ de los encuestados mencionaron que prefieren la carne y pollo a la brasa cuando asisten a un restaurante, mientras que el $20 \%$ seleccionaron que consumen comida criolla, el $19 \%$ prefieren comida rápida, en cambio los almuerzos saludables cuentan con un $17 \%$ de aceptación, el $8 \%$ y $9 \%$ prefiere ir a chifas o a un restaurante italiano, con lo que se demuestra que consumidores se inclinan mayormente por la carnes y pollo a la brasa, alimentos criollos y comidas rápidas esta sería una ventaja para la franquicia, pues La parrilla del Nato ofrece a su clientela estas tres líneas de alimentos.

3. ¿Cuántas veces al mes come en un restaurante? 


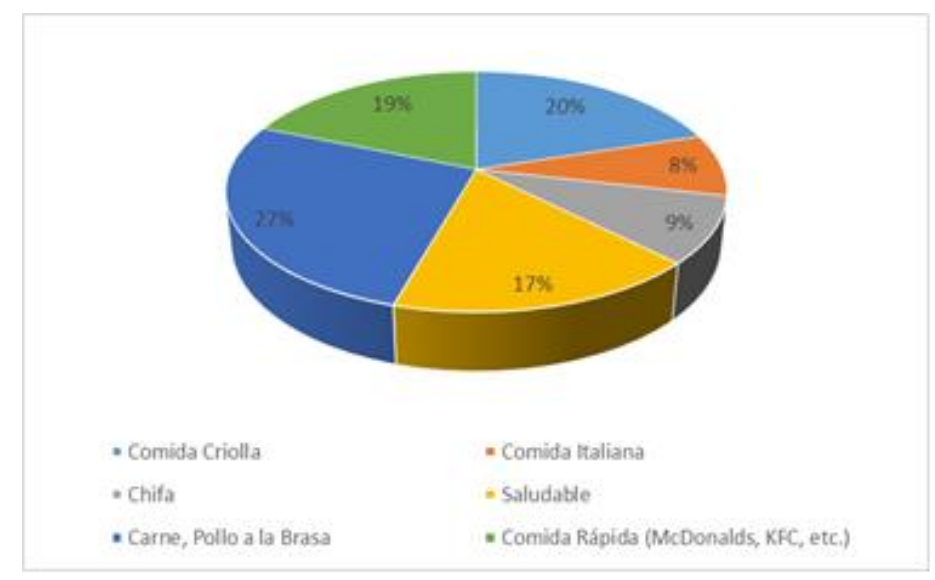

Figura 4. Frecuencia de consumo en un restaurante

Elaborado por: Ing. Gustavo Espinoza Delgado y Mgs. Pablo Ricardo San Andrés Reyes

El $63 \%$ de los encuestados mencionan que van 5 veces o más a un restaurante a la hora de su almuerzo, el 23\% mencionaron que asisten de 1-2 veces por semana, pues prefieren la comida casera y el $14 \%$ acude a un restaurante de 3-5 veces por semana. Esta pregunta genera dos indicadores, el primero que sí existe afluencia constante de clientes cerca al sector en el que se ubicará el restaurante, y el segundo es que el mejor horario para ofertar las parrilladas es en el receso de almuerzo.

4. ¿Por qué razón prefiere comer en un restaurante?

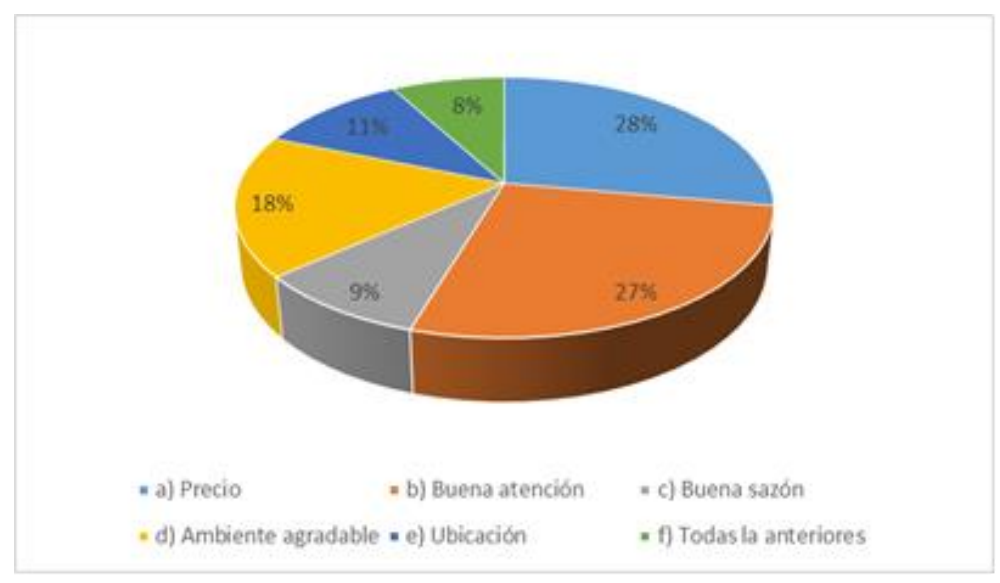

Figura 5. Gustos y preferencias del cliente

Elaborado por: Ing. Gustavo Espinoza Delgado y Mgs. Pablo Ricardo San Andrés Reyes

El $63 \%$ de los encuestados mencionaron que prefieren ir a un restaurante porque no tienen tiempo para cocinar, el $29 \%$ argumentaron que prefieren comer en casa y un $8 \%$ van a un restaurante por diversión. Esta pregunta demuestra que existe una necesidad que puede ser resuelta con el servicio de venta de alimentos que brindara La parrilla del Nato.

5. ¿Qué elementos influyen en la elección de un restaurante? 


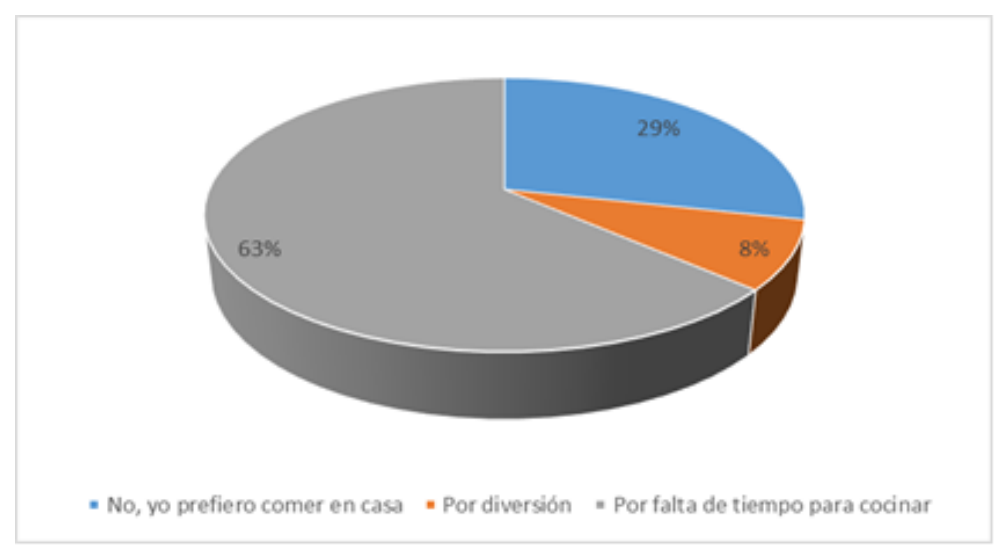

Figura 6. Causas que motivan a elegir un restaurante

Elaborado por: Ing. Gustavo Espinoza Delgado y Mgs. Pablo Ricardo San Andrés Reyes

El 28\% de los encuestados mencionan que al momento de elegir un restaurante optan por ver los precios de los platillos, mientras que el $27 \%$ dicen que prefieren la buena atención. Estos datos son muy relevantes para el estudio, pues ayudaría a elegir un precio promedio de los platillos y manejar personal calificado para atención al cliente, y de este modo cumplir con los gustos y preferencias de este segmento.

6. ¿Considera que La parrilla del Nato este localizado en el centro de Manta?

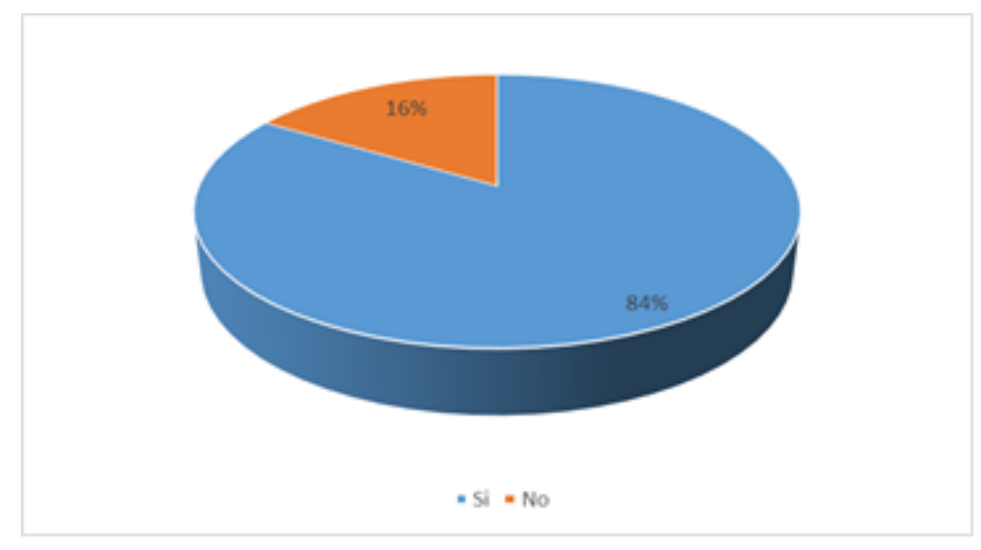

Figura 7. Lugar para localizar el local

Elaborado por: Ing. Gustavo Espinoza Delgado y Mgs. Pablo Ricardo San Andrés Reyes. Manta.

Se obtuvo una aceptación del $84 \%$ de que la organización se ubique en el centro de

\section{Conclusiones}

Se pudo determinar que, los alimentos son procesados de manera mixta artesanal y profesional, permitiendo crear variedad de platillos y bebidas con mano de obra calificada, además los precios con los que cuenta le permiten ser competitivos. Al no contar con sucursales centraliza la marca en un solo cantón y las políticas económicas pueden influenciar en el cambio 
de la inflación o en la tasa de desempleo con relación al negocio. Se diagnosticó que en Manta aún no se han determinado extensamente restaurantes que vendan especialmente comida a la parrilla al estilo argentino y que la competencia es "Media", pues a pesar que la "Parrilla del Nato" cuenta con platos a la parrilla únicos, existe algunos de lugares con productos similares.

A pesar de no tener mayores obstáculos de entrada a esta plaza, se estima que la carne, pollo, vegetales y demás ingredientes serán adquiridos en mercados como el Mercado Municipal de Manta, y otros supermercados de la ciudad. El poder de negociación de los consumidores en este caso es restringido, especialmente porque se brindan un servicio característico del resto restaurantes.

Se obtuvo un itinerario positivo para el estudio de caso, pues se demuestra que solos o acompañados, los encuestados acuden a un restaurante y que los consumidores se inclinan mayormente por las carnes y pollo a la brasa, comida criolla y comidas rápidas. Esto es una ventaja para la franquicia, pues "La parrilla del Ñato" ofrece a su clientela estas tres líneas de alimentos, adicionalmente se generaron dos indicadores, el primero que sí existe afluencia constante de clientes cerca al sector en el que se ubicará el restaurante, y el segundo es que el mejor horario para ofertar las parrilladas es en el receso de almuerzo. La mayoría de datos son muy relevantes para el estudio, pues ayudaría a elegir un precio promedio de los platillos y manejar personal calificado para atención al cliente, de este modo cumplir con los gustos y preferencias de este segmento. Es importante recalcar que un producto de calidad debe estar combinada por un servicio de excelencia y aceptación del precio en el mercado.

Por lo tanto, la investigación refleja la tendencia de expansión a través de franquicias del negocio "Parrilla del Ñato" hacia la ciudad de Manta, con aceptación de los potenciales posibles consumidores, demostrando que los negocios pymes en Ecuador pueden aplicar este modelo para su rápido crecimiento, expansión, generación de empleo y posicionamiento a nivel nacional para lograr la internacionalización a largo plazo.

\section{Bibliografía}

Alonso Prieto, M. (2012). Una franquicia para mí nuevo negocio. Editorial Selina Olmedo, Pág. 255.

Amorós, J. E., Gutiérrez, I., y Varela, C. (2011). Emprender desde la pequeña y mediana empresa: nueve casos de éxito de emprendedores latinoamericanos. Universidad de Desarrollo, Facultad de Economía y Negocios. Santiago, Chile, 137.

Arenas-Torrado, M. (2017). Franquicias: Oportunidad de Negocios Para la Competitividad de Restaurantes y Comidas Rápidas en Cúcuta. En Desarrollo Gerencial Revista de la Facultad de Ciencias Económicas Administrativas y Contables de la Universidad Simón BolívarColombia, 9(2), 158-173.

Arias, F. (2014). La administración y sus definiciones. México: Trillas.

Baena, G. (2010). "Modeling global franchising in emerging markets. An entry mode analysis". 
Journal of East West Business, Vol. 15, No.3, pp. 164-188.

Calomarde, J. V. (2013). Marketing Ecológico. Madrid: Ed. Pirámide ESIC.

Carrascal, Y. (16 de Enero de 2014). Estrategia Competitiva. Recuperado el 13 de Julio de 2016, de http://es.slideshare.net/YARIANYCARRASCAL/estrategiacompetitiva-30104913.

Castro Monge, E. (2010). Las estrategias competitivas y su importancia en La buena gestión de las empresas. Universidad de Oviedo-España, 28-No. 1; Pág. 247-276.

Cevallos Bravo, N. (2014). Estrategia competitiva. Guayaquil: Escuela Superior Politécnica del Litoral.

Chavarría López, L. R. (2013). "Las franquicias como una opoción de crecimiento para las microempresas: propuesta de un modelo”. México: Institución Politécnico Nacional.

Congo Suárez, M. d. (2016). “Análisis y Evaluación Económica-Financiera de la empresa LUMICAR, de la Ciudad de Loja, Periodo 2013 - 2014”. Loja - Ecuador: Universidad Nacional De Loja.

De Castro, E. y Cataluña, F, (2004). La investigación sobre franquicia. Investigaciones Europeas de Dirección Economía de la Empresa, 10(3), 71-96.

Édgar Emilio Salazar Baquero. (2011). Análisis de las implicaciones no financieras de la aplicación de la NIIF para PYME en las medianas entidades en Colombia. Bogotá: Cuadernos de Contabilidad.

El Economista. (20 de Julio de 2015). Las franquicias de distribución, en las que el franquiciado/da hace de. Recuperado el 13 de JUlio de 2015, de Las franquicias de distribución, en las que el franquiciado/da hace de: http://exportacionservicios.blogspot.com/2015/07/lafranquicia-tambien-entra-en-hogares.html

Enrich Cardona, R. (2013). Implantación de un sistema ERP SAP en una empresa. Barcelona España: Universidad Politécnica de Cataluña.

Fernandéz Sánchez, J. L. (2013). Introducción a la economía de la empresa. Cantabria - España: Universidad de Cantabria.

Fernandez, M. (2012). La Función Productiva De La Empresa. Mexico: riconomia.

Fernández, Z., y Nieto, M. (2005). La estrategia de internacionalización de la pequeña empresa familiar. Cuadernos de Economía y Dirección de la Empresa (22), 107-125. Madrid, España. Recuperado de http://www.redalyc.org/articulo.oa?id=80720287005

Guerrero, Armenteros, Acosta, \& Medina. (2014). Desarrollo Estratégico De Las 
Franquicias Mexicanas: Estudio Empírico En La Comarca Lagunera. México.: Universidad Autónoma de Coahuila, Vol. 7.

INEC. (2011). Población Economicamente Activa (Manta en cifras). Manta: https://lahora.com.ec/noticia/1000226824/la-ciudad-de-manta-en-cifras.

InfoFranquicias. (20 de Junio de 2016). Franquicias. Recuperado el 12 de Julio de 2016, de http://www.infofranquicias.com/cd-7138/Definicion-de-franquicias.aspx

Koontz. (2012). Estrategia, planificación y control. California: Mc Graw Hill.

La Hora. (2011). Microempresas, buena fuente de ingresos. Guayaquil-Ecuador: La hora nacional.

Mintzberg, H. (Diciembre de 2010). Estrategia empresarial. Recuperado el 13 de Julio de 2016, de Estrategia empresarial.: http://www.cynertiaconsulting.com/sites/default/files/PDF/Estrategia_empresarialcomo_formularla_e_implementarla_con_exito.pdf

Ñato. (2016). La Historia de tu Parrilada Preferida. Guayaquil: http://www.parrilladelnato.com/index.php/es/sobre-nosotros/historia.

Ordoñez, G. (2013). Planeacion estrategica; Guía de Administración. México: Azteca.

Parraga, C. (6 de 11 de 2011). Planeación estratégica direccionada al manejo de franquicias. Obtenido de http://dspace.ucuenca.edu.ec/bitstream/123456789/1057/1/tad1057.pdf

Rial Rebullido, T., \& Villanueva Lameiro, C. (2013). Análisis de la franquicia como modelo de gestión en el fitness. Revista Digital. , Año 17 - Pág. 168.

Rondán, F., Navarro, A., Díez de Castro, E., \& Rodríguez, R. (2010). "Estudios del Perfomance de los grupos estratégicos en el sistema de franquicias Español”. Sevilla - España: Universidad de Sevilla.

Solís, V., y González, M. (2007). La franquicia como estrategia de crecimiento de las pymes españolas. Conocimiento, innovación y emprendedores. La Rioja: FEDRA-Universidad de La Rioja, 2743-2756. Recuperado de https://dialnet.unirioja.es/descarga/articulo/2232656.pdf

Zambrano, E. (2010). Es la microempresa una solucion para el Ecuador. Visa al desarrollo.

Zamora Ramírez, J., \& Hiram Martell Torres, Y. (2014). Los sistemas de franquicias, estrategia viable para crecer en el mercado nacional e internacional. San Luis Potosí - México: Universidad Autónoma de San Luis Potosí.

Zapata, P. (2012). Contabilidad General, 6ta Edición. Editorial Mc Graw Hill. 